\title{
A Note on the p-Adic Interpolation Function for Multiple Generalized Genocchi Numbers
}

\author{
Serkan Araci ${ }^{1, *}$, Mehmet Acikgoz ${ }^{2}$, Erdoğan Şen $^{3}$ \\ ${ }^{1}$ Hatay, Turkey \\ ${ }^{2}$ Department of Mathematics, Faculty of Science and Arts, University of Gaziantep, Gaziantep, Turkey \\ ${ }^{3}$ Department of Mathematics, Faculty of Science and Letters, Namik Kemal University, Tekirdağ, Turkey \\ *Corresponding author: mtsrkn@hotmail.com
}

Received August 14, 2013; Revised September 17, 2013; Accepted September 25, 2013

\begin{abstract}
In the present paper, we deal with multiple generalized Genocchi numbers and polynomials. Also, we introduce analytic interpolating function for the multiple generalized Genocchi numbers attached to $\chi$ at negative integers in complex plane and we de.ne the multiple Genocchi p-adic L-function. Finally, we derive the value of the partial derivative of our multiple $p$-adic l-function at $\mathrm{s}=0$.
\end{abstract}

Keywords: multiple generalized Genocchi numbers and poly-nomials, Euler-Gamma function, p-adic interpolation function, multiple gen-eralized zeta function

Cite This Article: Serkan Araci, Mehmet Acikgoz, and Erdoğan Şen, "A Note on the P-Adic Interpolation Function for Multiple Generalized Genocchi Numbers." Turkish Journal of Analysis and Number Theory 1, no. 1 (2013): 17-22. doi: 10.12691/tjant-1-1-5.

\section{Preliminaries}

The works of generalized Bernoulli, Euler and Genocchi numbers and polynomials and their combinatorial relations have received much attention [1,8-32,36,37,38]. Generalized Bernoulli polynomials, generalized Euler polynomials and generalized Genocchi numbers and polynomials are the signs of very strong relationship between elementary number theory, complex analytic number theory, Homotopy theory (stable Homotopy groups of spheres), differential topol- ogy (differential structures on spheres), theory of modular forms (Eisenstein series), p-adic analytic numbers theory (p-adic L-functions), quantum physics(quantum Groups).

p-adic numbers also were invented by German Mathematician Kurt Hensel around the end of the nineteenth century. In spite of their being already one hundred years old, these numbers are still today enveloped in an aura of mystery within the scientific community. The p-adic integral was used in mathematical physics, for instance, the functional equation of the q-zeta function, qstirling numbers and q- Mahler theory of integration with respect to the ring $\mathbb{Z}_{\mathrm{P}}$ together with Iwasawa's P-adic $\mathrm{L}$ functions.

Also the p-adic interpolation functions of the Bernoulli and Euler polynomials have been treated by Tsumura [39]. Kim [11-34] also studied on p-adic inter- polation functions of these numbers and polynomials which are studied by many authors (see [3-43]). In the last decade, a surprising number of papers appeared proposing new generalizations of the Bernoulli, Euler and Genocchi polynomials to real and complex variables.

In [11-34], Kim studied some families of multiple Bernoulli, Euler and Genocchi numbers and polynomials. By using the fermionic p-adic invariant integral on $\mathbb{Z}_{\mathrm{P}}$, he constructed p-adic Bernoulli, p-adic Euler and p-adic Genocchi numbers and polynomials of higher order.

While many of the properties of Genocchi polynomials bear a close resemblance to the corresponding properties of Bernoulli and Euler polynomials, some properties are rather different. Obviously, Genocchi polynomials are worthy of an investigation perse.

In this paper, by using Kim's method in [28], we derive several properties for the multiple generalized Genocchi numbers attached to $\chi$.

In the complex plane, Genocchi numbers are defined in the complex plane by the generating function:

$$
C(t)=\frac{2 t}{e^{t}+1}=\sum_{n=0}^{\infty} G_{n} \frac{t^{n}}{n !}|t|<\pi
$$

It follows from the description that $G_{0}=0, G_{1}=1$, $G_{2}=-1, G_{3}=0, G_{4}=1, G_{5}=0, \cdots$, and $G_{2 k+1}=0$ for $k=1,2,3, \cdots$. (see $[2,3,4,7,12,13,16])$.

The Genocchi polynomials are also given by the rule:

$$
C(t, x)=e^{t G(x)}=\sum_{n=0}^{\infty} G_{n}(x) \frac{t^{n}}{n !}=\frac{2 t}{e^{t}+1} e^{x t}
$$

with the usual convention of replacing $G^{n}(x):=G_{n}(x)$ (see $[2,3,4,7,12,13,16])$. 
Let $w \in \mathbb{N}$. Then the multiple Genocchi polynomials of order $w$ are given by [13]

$$
C^{(w)}(t, x)=\left(\frac{2 t}{e^{t}+1}\right)^{w} e^{x t}=\sum_{n=0}^{\infty} G_{n}^{(w)}(x) \frac{t^{n}}{n !},|t|<\pi \quad(1.2)
$$

Taking $x=0.2$ in (1.2), then we have $G_{n}^{(w)}(0):=G_{n}^{(w)}$ are called the multiple Genocchi numbers of order $w$.

For $f \in \mathbb{N}$ with $\mathrm{f} \equiv 1(\bmod 2)$, we assume that $\chi$ is a primitive Dirichlet's charachter with conductor $\mathrm{f}$. It is known in [13] that the Genocchi numbers associated with $\chi, G_{n, \chi}$, was introduced by the following expression

$$
\begin{aligned}
C_{\chi}(t) & =2 t \sum_{\xi=1}^{f} \frac{\chi(\xi)(-1)^{\xi} e^{\xi t}}{e^{f t}+1} \\
& =\sum_{n=0}^{\infty} G_{n, \chi} \frac{t^{n}}{n !},|t|<\frac{\pi}{f}
\end{aligned}
$$

In this paper, we contemplate the definition of the generating function of the multiple generalized Genocchi numbers attached to $\chi$ in the complex plane. From this definition, we introduce an analytic interpolating function for the multiple generalized Genocchi numbers attached to $\chi$. Finally, we investigate behaviour of analytic interpolating function at $\mathrm{s}=0$.

\section{On an Analytic Function in Connection with the Multiple Generalized Genocchi Numbers}

In this part, we introduce the multiple generalized Genocci numbers attached to $\chi$ defined by

$$
\begin{aligned}
C_{\chi}^{(w)}(t) & =\sum_{n=0}^{\infty} G_{n, \chi}^{(w)} \frac{t^{n}}{n !} \\
& =(2 t)^{w} \sum_{a_{1}, \cdots, a_{w}=1}^{f} \frac{\left[\begin{array}{l}
(-1)^{a_{1}+\cdots+a_{w}} \\
e^{t\left(a_{1}+\cdots+a_{w}\right)}
\end{array}\left(a_{1}+\cdots+a_{w}\right)\right.}{\left(e^{f t}+1\right)^{w}}
\end{aligned}
$$

On account of (1.2) and (2.1), we easily derive the following

$$
\begin{aligned}
C_{n, \chi}^{(w)} & \frac{f^{n}}{f^{w}} \sum_{a_{1}, \cdots, a_{w}=1}^{f}\left[\begin{array}{l}
(-1)^{a_{1}+\cdots+a_{w}} \chi\left(a_{1}+\cdots+a_{w}\right) \\
G_{n}^{(w)}\left(\frac{a_{1}+\cdots+a_{w}}{f}\right)
\end{array}\right]
\end{aligned}
$$

For $s \in \mathbb{C}$, we have

$$
\begin{aligned}
& \frac{1}{\Gamma(s)} \int_{0}^{\infty} t^{s-w-1}\left\{(-1)^{w} C^{(w)}(-t, x)\right\} d t \\
= & 2^{w} \sum_{n_{1}, \cdots, n_{w} \geq 0} \frac{(-1)^{n_{1}+\cdots+n_{w}}}{\left(x+n_{1}+\cdots+n_{w}\right)^{S}}, x \neq 0,-1,-2, \cdots
\end{aligned}
$$

where $\Gamma(s)$ is Euler-Gamma function, which is de.ned by the rule

$$
\Gamma(s)=\int_{0}^{\infty} t^{s-1} e^{-t} d t
$$

Thanks to (2.3), we give the multiple Genocchi-zeta function as follows: for $s \in \mathbb{C}$ and $x \neq 0,-1,-2, \cdots$,

$$
\zeta_{G}^{(w)}(s, x)=2^{w} \sum_{n_{1}, \cdots, n_{w} \geq 0} \frac{(-1)^{n_{1}+\cdots+n_{w}}}{\left(x+n_{1}+\cdots+n_{w}\right)^{S}}
$$

By (1.2) and (2.3), we see that

$$
\zeta_{G}^{(w)}(-n, x)=\frac{G_{n+w}^{(w)}(x)}{\left(\begin{array}{c}
n+w \\
w
\end{array}\right) w !}
$$

for $n \in \mathbb{N}$.

By utilizing from complex integral and (2.1), we obtain the following equation: for $s \in \mathbb{C}$.

$$
\begin{aligned}
& \frac{1}{\Gamma(s)} \int_{0}^{\infty} t^{s-w-1}\left\{(-1)^{w} C_{\chi}^{(w)}(-t, x)\right\} d t \\
= & 2^{w} \sum_{\substack{n_{1}, \cdots, n_{w}=0 \\
n_{1}+\cdots+n_{w} \neq 0}} \frac{\chi\left(a_{1}+\cdots+a_{w}\right)(-1)^{n_{1}+\cdots+n_{w}}}{\left(n_{1}+\cdots+n_{w}\right)^{S}}
\end{aligned}
$$

where $\chi$ is the primitive Dirichlet's character with conductor

$$
f \in \mathbb{N} \text { and } f \equiv 1(\bmod 2)
$$

Because of (2.5), we give the de.nition Dirichlet's type of multiple Genocchi L-function in complex plane as follows:

$$
\begin{aligned}
& L^{(w)}(s \mid \chi) \\
& =2^{w} \sum_{\substack{n_{1}, \cdots, n_{w}=0 \\
n_{1}+\cdots+n_{w} \neq 0}}^{\infty} \frac{\chi\left(a_{1}+\cdots+a_{w}\right)(-1)^{n_{1}+\cdots+n_{w}}}{\left(n_{1}+\cdots+n_{w}\right)^{S}}
\end{aligned}
$$

Via the (2.1) and (2.6), we derive the following theorem: Theorem 1. For any $n \in \mathbb{N}$, then we have

$$
L^{(w)}(-n \mid \chi)=\frac{G_{n+w, \chi}^{(w)}(x)}{\left(\begin{array}{c}
n+w \\
w
\end{array}\right) w !}
$$

Let $\mathrm{s}$ be a complex variable, and let $\mathrm{a}$ and $\mathrm{b}$ be integer with $0<a<F$ and $F \equiv 1(\bmod 2)$

Thus, we can consider the partial zeta function $S^{(w)}$ $\left(s ; a_{1}, \cdots, a_{w} \mid F\right)$ as follows:

$$
\begin{aligned}
& S^{(w)}\left(s ; a_{1}, \cdots, a_{w} \mid F\right) \\
= & 2^{w} \sum_{\substack{m_{1}, \cdots, m_{w}>0 \\
m_{i} \equiv a_{i}(\bmod F)}} \frac{(-1)^{m_{1}+\cdots+m_{w}}}{\left(m_{1}+\cdots+m_{w}\right)^{S}} \\
= & (-1)^{a_{1}+\cdots+a_{w}} F^{-s} \zeta_{G}^{(w)}\left(s, \frac{a_{1}+\cdots+a_{w}}{F}\right)
\end{aligned}
$$

Theorem 2. The following identity holds: 


$$
\begin{aligned}
& S^{(w)}\left(s ; a_{1}, \cdots, a_{w} \mid F\right) \\
= & (-1)^{a_{1}+\cdots+a_{w}} F^{-s} \zeta_{G}^{(w)}\left(s, \frac{a_{1}+\cdots+a_{w}}{F}\right)
\end{aligned}
$$

Then Dirichlet's type of multiple L-function can be expressed as the sum: for $s \in \mathbb{C}$

$$
\begin{aligned}
& L^{(w)}(s \mid \chi) \\
= & \sum_{a_{1}, \cdots, a_{w}=1}^{F} \chi\left(a_{1}+\cdots+a_{w}\right) S^{(w)}\left(s ; a_{1}, \cdots, a_{w} \mid F\right)
\end{aligned}
$$

Substituting $s=w-n$ into (2.8), we readily derive the following: for $w, n \in \mathbb{N}$

$$
\begin{aligned}
& \left(\begin{array}{c}
n \\
m
\end{array}\right) w ! S^{(w)}\left(w-n ; a_{1}+\cdots+a_{w} \mid F\right) \\
= & (-1)^{a_{1}+\cdots+a_{w}} F^{n-w} G_{n}^{(w)}\left(\frac{a_{1}+\cdots+a_{w}}{F}\right)
\end{aligned}
$$

By (1.2), it is simple to indicate the following

$$
G_{n}^{(w)}(x)=\sum_{k=0}^{n}\left(\begin{array}{l}
n \\
k
\end{array}\right) x^{n-k} G_{n}^{(w)}=\sum_{k=0}^{n}\left(\begin{array}{l}
n \\
k
\end{array}\right) x^{k} G_{n-k}^{(w)}
$$

Thanks to (2.8), (2.10) and (2.11), we develop the following theorem:

Theorem 3. The following identity

$$
\begin{gathered}
\quad w !\left(\begin{array}{c}
-s \\
w
\end{array}\right) S^{(w)}\left(s+w ; a_{1}+\cdots+a_{w} \mid F\right) \\
=(-1)^{a_{1}+\cdots+a_{w}} F^{-w}\left(a_{1}+\cdots+a_{w}\right)^{-s} \\
\quad \times \sum_{k \geq 0}\left(\begin{array}{c}
-s \\
k
\end{array}\right)\left(\frac{F}{a_{1}+\cdots+a_{w}}\right)^{k} G_{k}^{(w)}
\end{gathered}
$$

is true.

From (2.9), (2.10) and (2.12), we have the following corollary:

Corollary 1. The following holds true:

$$
\begin{aligned}
& w !\left(\begin{array}{c}
-s \\
w
\end{array}\right) L^{(w)}(s+w \mid \chi) \\
= & \sum_{a_{1}, \cdots, a_{w}=1}^{F}\left[\begin{array}{l}
\chi\left(a_{1}+\cdots+a_{w}\right)(-1)^{a_{1}+\cdots+a_{w}} \\
F^{-w}\left(a_{1}+\cdots+a_{w}\right)^{-s}
\end{array}\right] \\
& \times \sum_{k=0}^{\infty}\left(\begin{array}{c}
-s \\
k
\end{array}\right)\left(\frac{F}{a_{1}+\cdots+a_{w}}\right)^{k} G_{k}^{(w)}
\end{aligned}
$$

The values of $L^{(w)}(s \mid \chi)$ at negative integers are algebraic, hence may be regarded as lying in an extension of $\mathbb{Q} p$. We therefore look for a p-adic function which agrees with $L^{(w)}(s \mid \chi)$ at the negative integers in the next section.

\section{Conclusion}

In this final section, we consider p-adic interpolation function of the multiple generalized Genocchi L-function, which interpolate Dirichlet.s type of multiple Genocchi numbers at negative integers. Firstly, Washington constructed $p$-adic $l$-function which interpolates generalized classical Bernoulli numbers.

Here, we use some the following notations, which will be useful in remainder of paper.

Let $\omega$ denote the Teichmüller character by the conductor $f_{\omega}=p$ For an arbitrary character $\chi$, we set $\chi_{n}=\chi \omega^{-n}, n \in \mathbb{Z}$, in the sense of product of characters. Let

$$
\langle a\rangle=\omega^{-1}(a) a=\frac{a}{\omega(a)}
$$

Thus, we note that $\langle a\rangle \equiv 1\left(\bmod p \mathbb{Z}_{p}\right)$. Let

$$
\begin{aligned}
& A_{j}(x)=\sum_{n=0}^{\infty} a_{n, j} x^{n}, \\
& a_{n, j} \in \mathbb{C}_{p}, j=0,1,2, \cdots
\end{aligned}
$$

be a sequence of power series, each convergent on a fixed subset

$$
T=\left\{\left.s \in \mathbb{C}_{p}|| s\right|_{p}<p^{-\frac{2-p}{p-1}}\right\}
$$

of $\mathbb{C}_{p}$ such that

(1) $a_{n, j} \rightarrow a_{n, 0}$ as $j \rightarrow \infty$ for any $n$;

(2) for each $s \in T$ and $\varepsilon>0$,there exists an $n_{0}=n_{0}(s, \varepsilon)$ such that

$$
\left|\sum_{n \geq n_{0}} a_{n, j} s^{n}\right|<\varepsilon \text { for } \forall j
$$

So,

$$
\lim _{j \rightarrow \infty} A_{j}(s)=A_{0}(s)
$$

for all $s \in T$.

This was firstly introduced by Washington [41] to indicate that each functions $\omega^{-s}(a) a^{s}$ and

$$
\sum_{k=0}^{\infty}\left(\begin{array}{l}
s \\
k
\end{array}\right)\left(\frac{F}{a}\right)^{k} B_{k}
$$

Where $F$ is multiple of $p$ and $f$ and $B_{k}$ is the $k$-th Bernoulli numbers, is analytic on $T$ (for more information, see [41]).

We assume that $\chi$ is a primitive Dirichlet's character with conductor $f \in \mathbb{N}$ with $f \equiv 1(\bmod 2)$. Then we contemplate the multiple Genocchi p-adic L-function, $L_{p}^{(w)} \equiv(s \mid \chi)$, which interpolates the multiple generalized Genocchi numbers attached to $\chi$ at negative integers.

For $f \in \mathbb{N}$ with $f \equiv 1(\bmod 2)$, let us assume that $F$ is a positive integral multiple of $p$ and $f=f_{\chi}$. We now give the definition of mutiple Genocchi p-adic L-function as follows: 


$$
\begin{aligned}
& w !\left(\begin{array}{c}
-s \\
w
\end{array}\right) L_{p}^{(w)}(s+w \mid \chi) \\
& =\frac{1}{F^{w}} \sum_{a_{1}, \cdots, a_{w}=1}^{F} \times(-1)^{a_{1}+\cdots+a_{w}} \\
& \times\left\langle a_{1}+\cdots+a_{w}\right\rangle^{-s} \\
& \times \sum_{k=0}^{\infty}\left(\begin{array}{c}
-s \\
k
\end{array}\right)\left(\frac{F}{a_{1}+\cdots+a_{w}}\right)^{k} G_{k}^{(w)}
\end{aligned}
$$

Due to (3.1), we want to note that $L_{p}^{(w)}(s+w \mid \chi)$ is an analytic function on $s \in T$.

For $n \in \mathbb{N}$, we have

$$
G_{n, \chi_{n}}^{(w)}=\frac{F^{n}}{F^{w}} \sum_{a_{1}, \cdots, a_{w}=1}^{F} \times{G_{n}}_{n}^{(w)}\left(\frac{a_{1}+\cdots+a_{w}}{F}\right)
$$

If $\chi_{n}(p) \neq 0$, then $\left(p, f_{\chi_{n}}\right)=1$, and so the ratio $\frac{F}{p}$ is a multiple $f_{\chi_{n}}$

Let

$$
\vartheta=\left\{\frac{a_{1}+\cdots+a_{w}}{p} \mid a_{1}+\cdots+a_{w} \equiv 0(\bmod p)\right\}
$$

for some $a_{i} \in \mathbb{Z}$ with $0 \leq a_{i} \leq F$.

Therefore we can write the following

$$
\begin{aligned}
& \frac{F^{n}}{F^{w}} \sum_{\substack{a_{1}, \cdots, a_{w}=1 \\
p \mid a_{1}+\cdots+a_{w}}} G_{n}^{(w)}\left(\frac{a_{1}+\cdots+a_{w}}{F}\right) \\
& \chi_{n}\left(a_{1}+\cdots+a_{w}\right) \\
& p^{n-w} \frac{\left(\frac{F}{p}\right)^{n}}{\left(\frac{F}{p}\right)^{w}} \chi_{n}(p) \\
& \quad \sum_{\substack{a_{1}, \cdots, a_{w}=1 \\
\lambda \in \vartheta}}^{\frac{F}{p}}(-1)^{\lambda} \chi_{n}(\lambda) G_{n}^{(w)}\left(\frac{\lambda}{F / p}\right)
\end{aligned}
$$

By (3.3), we de.ne the different multiple generalized Genocchi numbers attached to $\chi$ as follows:

$$
\begin{aligned}
& G_{n, \chi_{n}}^{*(w)} \\
& =\frac{\left(\frac{F}{p}\right)^{n}}{\left(\frac{F}{p}\right)^{w} \sum_{\substack{a_{1}, \cdots, a_{w}=1 \\
\lambda \in \vartheta}}^{\frac{F}{p}}(-1)^{\lambda} \chi_{n}(\lambda) G_{n}^{(w)}\left(\frac{\lambda}{F / p}\right)}
\end{aligned}
$$

On accounct of (3.2), (3.3) and (3.4), we attain the following

$$
\begin{gathered}
G_{n, \chi_{n}}^{(w)}-p^{n-w} \chi_{n}(p) G_{n, \chi_{n}}^{*(w)} \\
(-1)^{a_{1}+\cdots+a_{w}} \\
=\frac{F^{n}}{F^{w}} \sum_{\substack{a_{1}, \cdots, a_{w}=1 \\
p \nmid a_{1}+\cdots+a_{w}}}^{F} G_{n}^{(w)}\left(\frac{a_{1}+\cdots+a_{w}}{F}\right)
\end{gathered}
$$

By the definition of the multiple Genocchi polynomials of order $w$, we write the following

$$
\begin{aligned}
& G_{n}^{(w)}\left(\frac{a_{1}+\cdots+a_{w}}{F}\right) \\
= & F^{-n}\left(a_{1}+\cdots+a_{w}\right)^{n} \sum_{k=0}^{n}\left(\begin{array}{l}
n \\
k
\end{array}\right)\left(\frac{F}{a_{1}+\cdots+a_{w}}\right)^{k} G_{k}^{(w)}
\end{aligned}
$$

By (3.5) and (3.6), we have

$$
\begin{aligned}
& G_{n, \chi_{n}}^{(w)}-p^{n-w} \chi_{n}(p) G_{n, \chi_{n}}^{*(w)} \\
&= \frac{1}{F^{w}} \sum_{\substack{a_{1}, \cdots, a_{w}=1 \\
p \nmid a_{1}+\cdots+a_{w} \times\left(a_{1}+\cdots+a_{w}\right)^{n}}}^{F} \times \chi_{n}(-1)^{a_{1}+\cdots+a_{w}} \\
& \times \sum_{k=0}^{n}\left(\begin{array}{l}
n \\
k
\end{array}\right)\left(\frac{F}{a_{1}+\cdots+a_{w}}\right)^{k} G_{k}^{(w)}
\end{aligned}
$$

By (3.1) and (3.7), we readily see that

$$
\begin{aligned}
& w !\left(\begin{array}{c}
n \\
w
\end{array}\right) L_{p}^{(w)}(w-n \mid \chi) \\
& =\frac{1}{F^{w}} \sum_{a_{1}, \cdots, a_{w}=1}^{F} \times\left(a_{1}+\cdots+a_{w}\right)^{n} \\
& \quad \times \sum_{k=0}^{\infty}\left(\begin{array}{c}
n \\
k
\end{array}\right)\left(\frac{F}{a_{1}+\cdots+a_{w}}\right)^{a_{1}+\cdots+a_{w}} G_{k}^{(w)} \\
& =G_{n, \chi_{n}}^{(w)}-p^{n-w} \chi_{n}(p) G_{n, \chi_{n}}^{*(w)}
\end{aligned}
$$

Consequently, we arrive at the following theorem.

Theorem 4. The following nice identity holds true:

$$
\begin{aligned}
& w !\left(\begin{array}{c}
-s \\
w
\end{array}\right) L_{p}^{(w)}(s+w \mid \chi) \\
& =\frac{1}{F^{w}} \sum_{a_{1}, \cdots, a_{w}=1}^{F} \times(-1)^{a_{1}+\cdots+a_{w}} \\
& \times\left\langle a_{1}+\cdots+a_{w}\right\rangle^{-s} \\
& \times \sum_{k=0}^{\infty}\left(\begin{array}{c}
-s \\
k
\end{array}\right)\left(\frac{F}{a_{1}+\cdots+a_{w}}\right)^{k} G_{k}^{(w)}
\end{aligned}
$$

Thus $L_{p}^{(w)}(s+w \mid \chi)$ is an analytic function on $T$. Additionally, for each $n \in \mathbb{N}$, we procure the following:

$$
w !\left(\begin{array}{l}
n \\
w
\end{array}\right) L_{p}^{(w)}(w-n \mid \chi)=G_{n, \chi_{n}}^{(w)}-p^{n-w} \chi_{n}(p) G_{n, \chi_{n}}^{*(w)} .
$$


Using Taylor expansion at $\mathrm{s}=0$, we have

$$
\left(\begin{array}{c}
-s \\
k
\end{array}\right)=\frac{(-1)^{k}}{k} s+\cdots \text { if } k \geq 1
$$

Differentiating on both sides in (3.1), with respect to $\mathrm{s}$ at $\mathrm{s}=0$, we obtain the following corollary.

Theorem 5. Let $F$ be a positive integral multiple of $p$ and $f$. Then we have

$$
\begin{gathered}
\left.\frac{\partial}{\partial s}\left(\left(\begin{array}{c}
-s \\
w
\end{array}\right) L_{p}^{(w)}(s+w \mid \chi)\right)\right|_{s=0} \\
=\frac{(-1)^{w}}{w} L_{p}^{(w)}(w \mid \chi) \\
=\frac{1}{w ! F^{w}} \sum_{a_{1}, \cdots, a_{w}=1}^{F} \times(-1)^{a_{1}+\cdots+a_{w}} \\
+\frac{1}{\left.w ! a_{1}+\cdots+a_{w}, p\right)=1} \times\left(1-\log _{p}\left(a_{1}+\cdots+a_{w}\right)\right) \\
\sum_{\substack{a_{1}, \cdots, a_{w}=1 \\
\left(a_{1}+\cdots+a_{w}, p\right)=1}}^{F} \times(-1)^{a_{1}+\cdots+a_{w}} \\
\times \sum_{m=1}^{\infty} \frac{(-1)^{m}}{m}\left(\frac{F}{a_{1}+\cdots+a_{w}}\right)^{k} G_{k}^{(w)}
\end{gathered}
$$

where $\log _{p} x$ is the p-adic logarithm.

\section{References}

[1] F. Qi, Integral representations and properties of Stirling numbers of the first kind, Journal of Number Theory, Volume 133, issue 7 (July, 2013), p. 2307-2319.

[2] S. Araci, Novel identities for q-Genocchi numbers and polynomials, Journal of Function Spaces and Applications, Volume 2012, Article ID 214961, 13 pages.

[3] S. Araci, D. Erdal and J. J. Seo, A study on the fermionic p-adic qintegral representation on $\mathbb{Z p}$ associated with weighted qBernstein and q-Genocchi polynomials, Abstract and Applied Analysis, Volume 2011, Article ID 649248, 10 pages.

[4] S. Araci, J. J. Seo and D. Erdal, New construction weighted (h; q)Genocchi numbers and polynomials related to Zeta type functions, Discrete Dynamics in Nature and Society, Volume 2011, Article ID 487490, 7 pages.

[5] S. Araci, M. Acikgoz and E. Sen, On the extended Kim.s p-adic qdeformed fermionic integrals in the $\mathrm{p}$-adic integer ring, J. Number Theory (2013).

[6] S. Araci, M. Acikgoz and H. Jolany, On p-adic interpolating function associated with modified Dirichlet's type of twisted qEuler numbers and polynomials with weight alpha, Journal of Classical Analysis, Volume 2, Number 1 (2013), 35-48.

[7] S. Araci, M. Acikgoz, and K. H. Park, A note on the q-analogue of Kim's p-adic log gamma type functions associated with qextension of Genocchi and Euler numbers with weight alpha, Bull. Korean Math. Soc. 50 (2013), No. 2, pp. 583-588.

[8] E. Cetin, M. Acikgoz, I. N. Cangul and S. Araci, A note on the (h; q)-Zeta-type function with weight $\alpha$, Journal of Inequalities and Applications 2013, 2013:100.

[9] B. C. Kellner, On irregular prime power divisors of the Bernoulli numbers, Mathematics of Computation, Volume 76, Number 257, January 2007, Pages 405-441.

[10] D.V. Kruchinin and V. V. Kruchinin, Application of a composition of generating functions for obtaining explicit formulas of polynomials, Journal of Mathematical Analysis and Applications, Volume 404, Issue 1, 1 August 2013, Pages 161-171.
[11] T. Kim, On a p-adic interpolation function for the q-extension of the generalized Bernoulli polynomials and its derivative, Discrete Mathematics, 309 (2009) 1593-1602.

[12] T. Kim, On the q-extension of Euler and Genocchi numbers, J. Math. Anal. Appl. 326 (2007) 1458-1465.

[13] T. Kim, On the multiple q-Genocchi and Euler numbers, Russian $J$. Math. Phys. 15 (4) (2008) 481-486.

[14] T. Kim, p-adic q-integrals associated with Changhee-Barnes.qBernoulli polynomials, Integral Transforms Spec. Funct. 15 (2004) 415-420.

[15] T. Kim, Multiple p-adic L-function, Russ. J. Math. Phys. 13 (2006), no. 2, 151-157.

[16] T. Kim, A Note on the q-Genocchi numbers and polynomials, Journal of Inequalities and Applications Article ID 71452, 8 pages.

[17] T. Kim, q-Volkenborn integration, Russ. J. Math. phys. 9 (2002), 288-299.

[18] T. Kim, An invariant $\mathrm{p}$-adic $\mathrm{q}$-integrals on $\mathbb{Z} \mathrm{p}$, Applied Mathematics Letters, vol. 21 (2008), pp. 105-108.

[19] T. Kim, and J-S. Cho, A note on multiple Dirichlet.s q-L-function, Adv. Stud. Contemp. Math. (Kyungshang) 11 (2005), no. 1, 57-60.

[20] T. Kim, A note on the q-multiple zeta function, Adv. Stud. Contemp. Math. (Kyungshang) 8 (2004), no. 2, 111-113.

[21] T. Kim, Remark on the multiple Bernoulli numbers, Proc. Jangjeon Math. Soc. 6 (2003), no. 2, 185-192.

[22] T. Kim, q-Euler numbers and polynomials associated with p-adic q-integrals, J. Nonlinear Math. Phys., 14 (2007), no. 1, 15-27.

[23] T. Kim, New approach to q-Euler polynomials of higher order, Russ. J. Math. Phys., 17 (2010), no. 2, 218-225.

[24] T. Kim, Some identities on the q-Euler polynomials of higher order and q-Stirling numbers by the fermionic p-adic integral on $\mathbb{Z}$ p, Russ. J. Math. Phys., 16 (2009), no.4,484-491.

[25] T. Kim and S.H. Rim, On the twisted q-Euler numbers and polynomials associated with basic q-1-functions, Journal of Mathematical Analysis and Applications, vol. 336 (2007), no. 1, pp. 738-744.

[26] T. Kim, On p-adic q-1-functions and sums of powers, J. Math. Anal. Appl. (2006).

[27] T. Kim, On the analogs of Euler numbers and polynomials associated with $\mathrm{p}$-adic $\mathrm{q}$-integral on $\mathbb{Z} \mathrm{p}$ at $\mathrm{q}=-1, J$. Math. Anal. Appl. 331 (2) (2007) 779-792.

[28] T. Kim, On p-adic interpolating function for q-Euler numbers and its derivatives, J. Math. Anal. Appl. 339 (1) (2008) 598-608.

[29] T. Kim, q-Bernoulli numbers and polynomials associated with Gaussian binomial coed cients, Russ. J. Math. Phys. 15 (1) (2008) 51-57.

[30] T. Kim, Euler numbers and polynomials associated with zeta functions, Abstr. Appl. Anal. (2008) Art. ID 581582, 11 pp.

[31] T. Kim, Analytic continuation of multiple q-zeta functions and their values at negative integers, Russ. J. Math. Phys. 11 (1) (2004) 71-76.

[32] T. Kim, Barnes-type multiple q-zeta functions and q-Euler polynomials, J. Phys. A 43 (2010), no. 25, 255201, 11 pp.

[33] T. Kim, On Euler-Barnes multiple zeta functions, Russ. J. Math. Phys. 10 (2003), no. 3, 261-267.

[34] T. Kim, Non-Archimedean q-integrals associated with multiple Changhee q-Bernoulli polynomials, Russ. J. Math. Phys. 10 (2003), no. 1, 91-98.

[35] T. Kim, On a p-adic interpolation function for the multiple generalized Euler numbers and its derivatives. J. Comput. Anal. Appl. 14 (2012), no. 3, 410-416.

[36] G. D. Liu, Generating functions and generalized Euler numbers, Proc. Japan Acad. Ser. A Math. Sci., 84 (2008), 29-34.

[37] C. Frappier, Representation formulas for entire functions of exponential type and Generalized Bernoulli polynomials, $J$. Austral. Math. Soc. Ser., 64 (1998), No. 3, 307-316.

[38] H-S. Vandiver, On Generalizations of the Numbers of Bernoulli and Euler, Vol. 23, No. 10 (1937), 555-559.

[39] H. Tsumura, On a p-adic interpolation of the generalized Euler numbers and it applications, Tokyo J. Math. 10 (2) (1987) 281-293.

[40] K. Shiratani and S. Yamamoto, On a p-adic interpolation function for the Euler numbers and its derivatives, Mem. Fac. Sci. Kyushu Univ. Ser. A 39 (1) (1985) 113-125.

[41] L-C. Washington, Introduction to cyclotomic fields, Second edition, Springer-Verlag, 1997. 
[42] G. J. Fox, A method of Washington applied to the derivation of a two-variable p-adic L-function, Paci.c J. Math. 209 (2003), 31-40.
[43] J. Diamond, On the values of p-adic L-functions at positive integers, Acta Arith. 35 (1979), 223-237. 